\title{
EVALUACIÓN DEL DAÑO POR RETINIA ARIZONENSIS (HEINRICH) (LEPIDOPTERA: TORTRICIDAE), PARÁMETROS ESTRUCTURALES Y REGENERACIÓN NATURAL EN PINUS NELSONII (SHAW) Y P. CEMBROIDES (ZUCC.) EN MIQUIHUANA, TAMAULIPAS, MÉXICO
}

\author{
IRINEO FERNANDO-LUIS, ${ }^{1,2}{ }^{*}$ GERARDO SÁNCHEZ-RAMOS, ${ }^{1}$ \\ José Guadalupe MARTíneZ-ÁVALOS ${ }^{1}$ y Pedro REYES-CASTILLO ${ }^{3}$
}

\author{
${ }^{1}$ Universidad Autónoma de Tamaulipas, Instituto de Ecología Aplicada, Calle División del Golfo 356 Col. \\ Libertad, C.P. 87019, Cd. Victoria, Tamaulipas, México. \\ ${ }^{2}$ Centro de Bachillerato Tecnológico Agropecuario No. 272, Pedro J. Méndez No. 602 Ote. Esq. Feliciano \\ Montelongo. Zona Centro, C.P. 87980, Cd. Ocampo, Tamaulipas. \\ ${ }^{3}$ Instituto de Ecología, A. C. Carr. Antigua a Coatepec No. 351, El Haya 91070, Xalapa, Veracruz, México \\ <gsanchez@uat.edu.mx>.
}

Recibido: 20/10/2014; aceptado: 14/07/2015

Fernando-Luis, I., Sánchez-Ramos, G., Martínez-Ávalos, J. G. y Reyes-Castillo, P. 2015. Evaluación del daño por Retinia arizonensis (Heinrich) (Lepidoptera: Tortricidae), parámetros estructurales y regeneración natural en Pinus nelsonii (Shaw) y $P$. cembroides (Zucc.) en Miquihuana, Tamaulipas, México. Acta Zoológica Mexicana (n.s.), 31(3): 367-379.

RESUMEN. El presente trabajo tuvo como objetivo determinar la intensidad del daño de Retinia arizonensis y su posible relación con algunos parámetros estructurales: diámetro de tallo, altura y cobertura, así como con la regeneración natural de los pinos piñoneros $P i$ nus cembroides y $P$. nelsonii. Para su evaluación se ubicaron sitios de 2,500 $\mathrm{m}^{2}$ de cada especie; en $P$. cembroides se contabilizaron 136 individuos de los cuales 135 presentaron un daño promedio de $10.5 \%$ ( \pm 8.1 D.E.) en brotes terminales. En P. nelsonii de un total de 47 individuos encontrados, 46 exhibieron daños por $R$. arizonensis, con un promedio de $4.8 \%$ ( \pm 4.5 D.E.), ca 2 veces menos que en $P$. cembroides. Al realizar la prueba de Kruskal-Wallis $\left(H-\mathrm{Chi}^{2}\right)$ del porcentaje de daño de $R$. arizonensis entre las especies, se observó marcada diferencia significativa $(p<0.001)$. Referente a la estructura, $P$. cembroides presentó los siguientes valores promedio: diámetro del tallo $=10.7$ $\mathrm{cm}( \pm 8.0$ D.E. $)$, altura $=2.9 \mathrm{~m}( \pm 1.0$ D.E. $)$ y cobertura $=3.0 \mathrm{~m}^{2}( \pm 1.8$ D.E.). En relación a $P$. nelsonii, los valores promedio fueron: diámetro del tallo $=10.7 \mathrm{~cm}( \pm 4.3)$, altura $=3.6 \mathrm{~m}( \pm 0.8)$ y cobertura $=4.2$ $\mathrm{m}^{2}( \pm 1.8)$, estas variables mostraron una amplia diferencia estadística $(p<0.001)$. Al medir la asociación del daño por $R$. arizonensis con las variables estructurales, $P$. cembroides mostró correlación positiva marginal con las variables altura, diámetro y cobertura con $r^{2}=0.12$, 0.16 y 0.18 , respectivamente $(p<0.001)$. Sin embargo, $P$. nelsonii no manifestó correlación significativa entre el daño por $R$. arizonensis con los parámetros estructurales. La influencia conjunta de las tres variables estructurales (correlación múltiple $r^{2}$ ) en el daño de $R$. arizonensis, quedó mayormente explicada en $P$. cembroides con valor de $r^{2}$ de 0.19 como altamente significativa $(p<0.001)$. Para $P$. nelsonii la influencia de las variables estructurales sobre el porcentaje de daño de $R$. arizonensis no fue significativa $(p=0.128)$. La regeneración presentó una participación porcentual (con respecto a los adultos) de $20.6 \%$ para $P$. cembroides y de $42.5 \%$ para $P$. nelsonii, con altura promedio de $13.9 \mathrm{~cm}( \pm 12.6)$ y $28.4 \mathrm{~cm}( \pm 20.0)$ en $P$. cembroides y $P$. nelsonii, respectivamente.

Palabras clave: Retinia arizonensis, $P$. cembroides y $P$. nelsonii, estructura y regeneración natural.
Fernando-Luis, I., Sánchez-Ramos, G., Martínez-Ávalos, J. G. y Reyes-Castillo, P. 2015. Damage evaluation of (Lepidoptera: Tortricidae) (Heinrich), structural parameters and natural regeneration in Pinus nelsonii (Shaw) and P. cembroides ( Zucc.) in Miquihuana, Tamaulipas. Mexico. Acta Zoológica Mexicana (n. s.), 31(3): 367-379.

ABSTRACT. The present study aims to determine the damage intensity of the pinyon pitch nodule moth, Retinia arizonensis and their possible relationship to some structural parameters: stem diameter, height and coverage, as well as the natural regeneration of Pinus cembroides and $P$. nelsonii. For their evaluation, sites with $2,500 \mathrm{~m}^{2}$ of each species were located. A total of 136 individuals of $P$. cembroides were recorded, with an average damage of $10.5 \%$ ( \pm 8.1 SD) in the terminal buds. In P. nelsonii, a total of 47 individuals were found, 46 exhibited damage from $R$. arizonensis, averaging $4.8 \%$ ( $\pm 4.5 \mathrm{SD})$, twice less than $P$. cembroides. We used the Kruskal-Wallis $\left(H-C h i^{2}\right)$ to assess the damage of $R$. arizonensis between species, marked significant difference $(p<0.001)$ was observed. Relating to the structure, $P$. cembroides showed the following mean values: stem diameter $=10.7 \mathrm{~cm}( \pm 8.0$ $\mathrm{SD})$, height $=2.9 \mathrm{~m}( \pm 1.0 \mathrm{SD})$ and coverage $=3.0 \mathrm{~m}^{2}( \pm 1.8 \mathrm{SD})$. With respect to $P$. nelsonii, these were: stem diameter $=10.7 \mathrm{~cm}( \pm 4.3)$, height $=3.6 \mathrm{~m}( \pm 0.8)$ and coverage $\mathrm{m}^{2}=4.2( \pm 1.8)$, these variables showed wide statistical differences $(p<0.001)$. When measuring the association of $R$. arizonensis damage, with the structural variables, $P$. cembroides showed a positive marginal correlation with the variables height, diameter and covering with $r^{2}=0.12,0.16$ and 0.18 , respectively $(p<0.001)$. However, $P$. nelsonii expressed non-significant correlations among $R$. arizonensis damage with the structural parameters. The combined influence of the three structural variables (multiple correlation $r^{2}$ ) of $R$. arizonensis damage, was mostly explained in P. cembroides with $r^{2}$ value of 0.19 highly significant $(p<0.001)$. In relation to $P$. nelsonii, the structural variables influenced on damage percentage of $R$. arizonensis were not significant ( $p=0.128$ ). Natural regeneration showed percentage participation (relative to adult trees) of $20.6 \%$ for $P$. cembroides and $42.5 \%$ for $P$. nelsonii with mean height of 13.9 $\mathrm{cm}( \pm 12.6)$ and $28.4 \mathrm{~cm}( \pm 20.0)$ in P. cembroides and P. nelsonii, respectively.

Key words: Retinia arizonensis, $P$. cembroides, $P$. nelsonii, structure and natural regeneration. 


\section{INTRODUCCIÓN}

Las tres especies de pinos piñoneros presentes en Tamaulipas (Pinus cembroides, $P$. nelsonii y P. pinceana Gordon), se distribuyen en un gradiente altitudinal que oscila de 1,150 a 2,700 m snm (Sánchez-Ramos \& Ledezma 1995, Sánchez-Ramos et al. 2005, Sánchez-Castillo 2013). Pinus cembroides (sección Parrya, subsección Cembroides) es de amplia distribución, en numerosos cerros y mesetas de la Sierra Madre Oriental, desde el estado de Puebla hasta los estados de Coahuila, Nuevo León y Tamaulipas, México, incluso se extiende hasta la frontera sur de los Estados Unidos de América (Perry 1991, Gernandt et al. 2003). Se distribuye en forma discreta y ocupa un área de ca 140,000 hectáreas en Tamaulipas (91\% del área total reportada para los bosques piñoneros), ocupando en esta zona el piso altitudinal de $\sim 1,700$ a 2,100 m snm en un gradiente Norte-Sur, donde es más común a medida que desciende (Sánchez-Ramos et al. 2005).

Por otra parte, Pinus nelsonii (sección Parrya, subsección Nelsoniae) está catalogada como especie en peligro de extinción por la Norma Oficial Mexicana NOM-059SEMARNAT-2010 (DOF 2010) y como vulnerable según criterios de la Unión Internacional para la Conservación de la Naturaleza (UICN 2011); su distribución se restringe en los estados del noreste de México como sigue: i) San Lorenzo, Aramberri, Galeana y Doctor Arroyo en Nuevo León, ii) Sierra del Carmen en Coahuila, iii) Las Tablas en San Luis Potosí y, iv) Miquihuana, Palmillas y Tula en Tamaulipas (Perry 1991).

Las poblaciones de los pinos piñoneros están expuestas a una serie de factores negativos, tanto de origen natural, como antrópico, tales como: i) incendios, ii) desmontes para la agricultura, iii) sobrepastoreo, iv) plagas y enfermedades, entre otros, provocando fragilidad y susceptibilidad al ataque de insectos así como a la fragmentación y disminución constante de la superficie boscosa (Challenger 1998, Martin et al. 2006). Posterior a la perturbación y en etapas de sucesión temprana, la diversidad de especies de árboles disminuye con relación a los bosques antiguos (RamírezMarcial et al. 2001), lo que conlleva hacia una pérdida de los recursos disponibles para el suelo (Caribello 2003).

Por ejemplo, en 1998 ocurrió una alta incidencia de incendios forestales en México, afectando una superficie de 849,633 ha, de las cuales 17,860 ha correspondieron a Tamaulipas. Posteriormente, en el año 2011 el fuego afectó 956,405 a nivel nacional, correspondiendo a Tamaulipas 15,958 hectáreas, dañando ca 4\% del arbolado adulto de encinos y pinos en los municipios de: Miquihuana, Bustamante y Jaumave, Tamaulipas (Conafor 2013). En 2013, en Miquihuana el fuego dañó 250 ha de matorral xerófilo y 50 ha de bosque de pino (Seduma 2013).
Las plagas y enfermedades han mermado grandes superficies de bosques de pino y encino en Tamaulipas (Sánchez-Ramos et al. 2005). Los insectos herbívoros (principalmente larvas de Lepidoptera) pueden considerarse como uno de los grupos de mayor representatividad e impacto en las comunidades ya que constituyen ca $90 \%$ de los defoliadores de bosques; dentro de este Orden, la familia Tortricidae se ubica entre las cuatro primeras en abundancia y diversidad (Marquis \& Whelan 1994, Wold $\&$ Marquis 1997). Se estima que la intensidad y la ocurrencia del daño dependen de varios factores inherentes del hospedante, tales como el diámetro del tallo (Jactel et al. 1996 y 2002), la edad (Maleque et al. 2010), la condición del vigor/estrés (Wenninger \& Inouye 2008) y la presencia de compuestos volátiles químicos atrayentes o defensivos (Hanks 1999, Jactel et al. 2002). Estos compuestos, son producidos principalmente en las flores, pero también se encuentran en los frutos, tallos, hojas y raíces (Steeghs et al. 2004). Los árboles de mayor dimensión, son más fáciles de ser detectados por los herbívoros que los más pequeños, según “per se size hypothesis” y la Teoría de la Apariencia (Feeny 1975 y 1976, Lawton 1983); cuando la concentración es mayor (e.g. follaje, partes vegetativas), se puede facilitar el encuentro y/o acceso de los herbívoros consumidores de follaje (Root 1973, Haddad 2001).

En México existen pocos estudios sobre la evaluación de la sanidad de los pinos piñoneros causados por insectos herbívoros, entre ellos se encuentran: i) la evaluación del daño de las principales plagas de conos y semillas de piñón en el Estado de Hidalgo (Martínez et al. 1985), ii) insectos que afectan conos y semillas en Baja California (Díaz-Ortiz 1988), iii) la evaluación de la mortalidad de conos y semillas en especies de piñoneros en Coahuila (Flores \& Díaz 1991), iv) la caracterización de daños causados por Retinia arizonensis en Nuevo León e Hidalgo (Cibrián 1985) y $v$ ) la evaluación del daño causado por $R$. arizonensis (Lepidoptera:Torticidae) en $P$. cembroides en Saltillo, Coahuila (Domínguez 2003), entre otros.

En el municipio de Miquihuana, Tamaulipas se han efectuado algunos estudios referentes a las plagas, diversidad de entomofauna y factores de mortalidad sobre conos y semillas en P. cembroides y P. nelsonii (Sánchez-Ramos et al. 1987, Ledezma et al. 1991, Sánchez-Ramos et al. 1991, Ruiz et al. 1997 a, b, Sánchez-Ramos et al. 2005). De lo registrado en daños para $P$. nelsonii, destaca el provocado por la chinche semillera (Leptoglossus occidentalis Heidemann), causando ca $66 \%$ de mortalidad, seguida por Dioryctria abietella (D. \& S.) con 20\% y Eucosma sp. con 13\% (Sánchez-Ramos \& Ledezma 1995). Para $P$. cembroides, el mayor daño también es causado por $L$. occidentalis (45\%), seguido por Conophthorus cembroides (Wood) con $11 \%$, otro $11 \%$ de mortalidad lo generan 
Eucosma bobana (Kearfott), Dioryctria sp., Contarinia sp. y factores mecánicos (e.g. caída de ramas), con un total de 67.2\% de mortalidad (Sánchez-Ramos et al. 2005); cifra similar, aunque calculada con diferentes factores de mortalidad, es reportada por Flores y Díaz (1991) para Saltillo, Coahuila.

En Tamaulipas, aun cuando existen datos sobre la distribución potencial de Retinia arizonensis (Cibrián et al. 1995, Ruiz et al. 1997 b) para la región de Miquihuana, no hay registros en la literatura especializada sobre el nivel daño que causa $R$. arizonensis sobre pinos piñoneros. Sin embargo, durante el periodo septiembre-noviembre del año 2012, se presentaron daños de $R$. arizonensis sobre los brotes terminales de las dos especies piñoneras: Pinus cembroides y P. nelsonii (Fernando-Luis \& Sánchez-Ramos, obs. pers.). Generando con ello, la necesidad de conocer la dinámica de la interacción ecológica planta-herbívoro y el efecto que la herbivoría causa sobre los pinos piñoneros.

Retinia arizonensis es una palomilla Lepidoptera de la familia Tortricidae, cuyo daño consiste en barrenar brotes y conos. El adulto deposita de 1 a 3 huevecillos en la base de las brácteas aciculares del brote en crecimiento (Cibrián et al. 1995), al emerger la larva se alimenta del cambium formando galerías (sin daño aparente). El daño se observa a principios de otoño, cuando las ramillas mueren y dan una apariencia de bandera roja (Cranshaw et al. 2000, Jacobi \& Cranshaw 2009). Las larvas son de rojizo-amarillento con cabeza bien desarrollada. Durante el invierno se forma una cámara de resina donde entra en diapausa, reiniciando su actividad en primavera. La pupación ocurre a finales de abril a junio, en una bolsa de seda en la parte más alta de la masa de resina para facilitar la emergencia del adulto. Se presenta una generación por año (Cibrián 1985, Jacobi \& Cranshaw 2009). El adulto es pequeño, sus alas anteriores miden de 15 a $20 \mathrm{~mm}$ con franjas irregulares de colores grises y café claro, con tonos iridiscentes; las anteriores son café grisáceo muy obscuro (Cibrián 1985). Los hospedantes más comunes son Pinus nelsonii, P. pinceana, P. cembroides, P. johannis, $P$. culminicula, $P$. edulis y P. remota (Flores \& Caldera 1985, Flores \& Martínez 1987).

Un aspecto elemental de los bosques es la estructura, la cual incluye la variedad de disposiciones o arreglos individuales (vertical y horizontal) y espaciales. En lo individual, se consideran registros de diversos tamaños y condiciones de árboles y tallos. En lo espacial, se refiere a la disposición de estos individuos, es decir, si están uniformemente espaciados o agrupados (Franklin et al. 2002, Franklin \& Van 2004). Estas características estructurales de los bosques, cada vez son más reconocidas y estudiadas por teóricos y prácticos (Franklin et al. 2002), ya que es un conocimiento básico para el desarrollo adecuado de planes de manejo (Jiménez et al. 2001, Urbieta 2009), para mantener la sanidad e implementar medidas, para reducir la susceptibilidad a los incendios (Perry et al. 2004), entre otras aplicaciones. En Miquihuana, se han realizado algunos trabajos sobre este aspecto, tales como: i) la estructura y composición florística asociada (Basáñez-Muñoz 1983), ii) su ecología y distribución (Suzán 1985 y 1987, Suzán \& Galarza 1987, Suzán \& SánchezRamos 1989), iii) la utilización y el manejo (Suzán et al. 1991), iv) la estimación de la estructura de un bosque de P. nelsonii en Tamaulipas (Suzán 1990) y, v) la estructura de la población $P$. cembroides y P. nelsonii (Suzán et al. 2001) pero se carece de estudios concernientes a la asociación de los herbívoros con respecto a la estructura de estas especies, mismos que permitirán reconocer las características ecológicas y adaptativas de ambos interactuantes.

Se entiende la regeneración natural como el proceso de reemplazo del arbolado maduro por nuevos individuos en una unidad de espacio y tiempo (Gómez-Pompa \& Ludlow 1976, Martínez-Ramos 1994 y 1995). Esta etapa regenerativa puede ocurrir a través de dos procesos: $i$ ) por germinación de semillas y ii) a través de rebrotes desde el tocón o raíz que emergen cuando las plantas existentes son cortadas, quemadas o derribadas (Harper 1977). Para el primero, es fundamental la participación de ciertos componentes de la comunidad, como son: lluvia, banco y dispersión de semillas (Mostacedo \& Pinard 2001, Paz \& Martínez-Ramos 2003, Martínez-Ramos \& GarcíaOrth 2007). Para ambos procesos (semillas y rebrotes), resultan imprescindibles los factores del medio físico, tales como: la humedad, fertilidad y acidez del suelo, la intensidad lumínica y el espesor de la capa del mantillo vegetal (Mostacedo \& Pinard 2001, Pérez-Ramos 2007, Queenborough et al. 2007). En la región de estudio, la regeneración natural de los bosques es considerada como crítica para la sucesión y el éxito reproductivo de las especies piñoneras (Suzán et al. 2001, Sánchez-Ramos et al. 2005). Un estudio previo realizado en Miquihuana, Tamaulipas, sobre el tema (densidad de plántulas presentes en 2,500 $\mathrm{m}^{2}$ ), demostró que la regeneración porcentual fue de $49.6 \%$ para $P$. cembroides y $63.4 \%$ para $P$. nelsonii (Fernando-Luis et al. 2012). Finalmente, los cambios en los patrones de precipitación y temperatura asociados con el cambio climático global, pueden afectar la producción de semillas y la regeneración natural de estas especies, ya que las altas temperaturas de verano reducen la supervivencia de las plántulas (Calama \& Montero 2007).

En adición a lo anterior, los efectos del cambio climático a través de la alteración de carbono, agua e intercambios de energía de la superficie de la tierra (Jentsch et al. 
2007, Adams et al. 2009), derivan en un incremento de la temperatura y con ello en la mayor presencia de insectos herbívoros (Menéndez 2007, Currano et al. 2008, DeLucia et al. 2008, Mitton \& Ferrenberg 2012). Esta compleja situación ubica a las poblaciones de especies piñoneras en un futuro incierto. Por tanto, se requieren estudios sucesivos sobre diversos tópicos, tales como: $i$ ) la dinámica de la interacción ecológica planta-herbívoro, ii) el efecto de los parámetros estructurales sobre el herbívoro y, iii) el estado de la regeneración de las especies piñoneras.

El presente estudio, tuvo como objetivo central determinar la intensidad del daño de Retinia arizonensis y su posible relación con tres parámetros estructurales (diámetro de tallo, altura y cobertura), así como con la regeneración natural de $P$. cembroides y $P$. nelsonii, en la región piñonera de Miquihuana, Tamaulipas. Una aplicación práctica de estos resultados, es prevenir la expansión de daños en ambas especies, para advertir a las autoridades fitosanitarias del municipio y del estado sobre el mantenimiento del ecosistema, en términos del funcionamiento y la sanidad.

\section{MATERIAL Y MÉTODOS}

Descripción del área de estudio. La investigación se desarrolló durante Septiembre del 2012 en el Municipio de Miquihuana, Tamaulipas, situado en la región norte de la Sierra Madre Oriental entre los paralelos 23 $47^{\prime}$ y $23^{\circ}$ 24' de latitud Norte y los meridianos $99^{\circ} 58^{\prime}$ y $99^{\circ} 35^{\prime}$ de longitud Oeste, con una altitud entre 1,300 y 3,600 m snm. El uso potencial del suelo y la vegetación está representado por: agricultura $8.4 \%$, zona urbana $0.2 \%$, bosque $62.4 \%$, matorral $27 \%$ y pastizal $2 \%$ (INEGI 2009). Los suelos son relativamente homogéneos y pobres en materia orgánica (6.5\%) con profundidad $<50 \mathrm{~cm}$ y pH entre 6.88.0 (Suzán 1990).

El bosque piñonero se encuentra en un clima Bsohwx, seco semicálido (García 1981), con una precipitación promedio anual de $362.6 \mathrm{~mm}$. Esta zona se caracteriza por la presencia de temperaturas extremas, la máxima llega a alcanzar los $41{ }^{\circ} \mathrm{C}$ en el mes de mayo y la mínima de -5 ${ }^{\circ} \mathrm{C}$ durante el periodo diciembre-febrero (Sánchez-Ramos \& Ledezma 1995). La vegetación asociada al bosque piñonero es el matorral rosetófilo (Basáñez-Muñoz 1983, Suzán 1990) con dominancia de los siguientes elementos: Juniperus flaccida Schlechtendal, J. monosperma (Engelm.) Sarg. (Cupresaceae); Dasylirion texanum Scheele, Agave lecheguilla Torr. (Agavaceae); Rhus pachyrrachis Hemsl., R. virens Lindh. ex A. Gray. (Anacardiaceae); Tillandsia recurvata (L.) (Bromeliaceae); Opuntia stenopetala Engelm., O. rastrera F.A.C.Weber, O. tunica- ta (Lehm.) F.M.Knuth, Thelocactus conothelos (Regel \& Klein) Backeb, Echinocactus platyacanthus Link \& Otto, Ferocactus hamatacanthus (Muehlenpf.) Britton \& Rose, Turbinicarpus pseudopectinatus (Backeb.) Glass \& R.C.Foster, T. beguinii (Taylor) Mosco \& Zanovello (1997) (Cactaceae); Heliotropium fruticulosum Herb Linn (Boraginaceae); Asclepias linaria Cav. (Asclepidaceae); Baccharis ramulosa (DC.) Gray., Brickellia veronicaefolia (Kunth) A. Gray., Chrysactinia mexicana A. Gray., Eupatorium espinosarum A. Gray., Taraxacum officinale G. H. Weber ex Wigg. (Compositae); Quercus pringlei Seemen, Q. microphylla Née (Fagaceae) y Acacia berlandieri Benth. (Fabaceae).

Muestreo. Se establecieron dos sitios con daño aparente de $R$. arizonensis (Pc: Pinus cembroides y Pn: Pinus nelsonii), cada uno comprendió un total de cuatro cuadrantes de $25 \times 25 \mathrm{~m}\left(2,500 \mathrm{~m}^{2}\right.$, en total $\left.5,000 \mathrm{~m}^{2}\right)$, localizados en el paralelo $23^{\circ} 32^{\prime}$ de latitud Norte y el meridiano $99^{\circ}$ $48^{\prime}$ de longitud Oeste; con una altitud entre 1,860 y 1,981 $\mathrm{m} \mathrm{snm}$. El muestreo realizado fue subjetivo (Samo et al. 2008), ya que se consideró a partir de la presencia de $R$. arizonensis en $P$. cembroides y $P$. nelsonii en la mencionada región, y que significara $>10 \%$ de la superficie de daño, para ambas especies.

Daño por Retinia arizonensis. Se realizó un recorrido en cada uno de los cuadrantes, cuantificando el daño visualmente (\% de daño/árbol), utilizando una retícula óptica transparente (acetato cuadriculado de $28 \times 21 \mathrm{~cm}$, con cuadrícula de $1 \mathrm{~cm}^{2}$ ), se observaron las copas de los árboles desde una distancia de $5 \mathrm{~m}$ con cuatro puntos de observación (puntos cardinales), cubriendo la totalidad de cada cuadrante y de cada especie. A cada individuo se le asignó el porcentaje de daño (promedio de las cuatro lecturas), de acuerdo a la cubierta porcentual de la retícula con yemas terminales y ramillas dañadas por el insecto (metodología tomada de Sánchez-Ramos \& Dirzo 2005 y modificada para el presente proyecto).

Para evaluar estadísticamente el daño por Retinia arizonensis se uso la Prueba estadística de Kruskal-Wallis $\left(H-C h i^{2}\right)$, utilizando el programa estadístico PAST versión 2.15 (Hammer et al. 2001), con la finalidad de determinar las diferencias dentro y entre las especies. Adicionalmente, el porcentaje de daño se agrupó en siete clases (véase Cuadro 2). Para determinar este número de clases $(K)$, se utilizó la fórmula propuesta por Sturges (1926):

$$
K=1+3.322 * \log n
$$

donde " $n$ " es igual al número de observaciones.

Densidad y estructura. En cada parcela se levantó un censo de $P$. cembroides y $P$. nelsonii, considerando a los individuos con diámetros $\geq 2 \mathrm{~cm}$ y con una altura de 80 
cm sobre el ras del suelo. Paralelo al conteo de plantas, se midió el diámetro de tallo, la altura de planta y la cobertura. El diámetro se midió con una forcípula Haglöff, para la altura se consideraron las plantas $<2 \mathrm{~m}$ (medidas directamente con un flexómetro Stanley), las de altura superior fueron medidas con un clinómetro electrónico Haglöff (Mostacedo \& Fredericksen 2000). La cobertura, se midió con un densiómetro cóncavo de acuerdo a Mostacedo y Fredericksen (2000). Además, los diámetros del tallo se agruparon en clases, utilizando la fórmula anterior.

Asociación de variables estructurales con el porcentaje de daño por Retinia arizonensis. Una vez evaluado el porcentaje de daño por $R$. arizonensis y las variables diámetro, altura y cobertura, se determinó el grado de asociación de las tres variables estructurales (en forma individual), utilizando el porcentaje de daño de $R$. arizonensis, a través del coeficiente de determinación $\left(r^{2}\right)$ y la influencia conjunta de las tres variables estructurales con el daño de $R$. arizonensis mediante una correlación múltiple $\left(r^{2}\right)$. Ambas determinaciones se realizaron con el Software STATISTICA version 6 (StatSoft Inc. 2004).

Regeneración: Para evaluar la regeneración natural, se

Cuadro 1. Daño causado por Retinia arizonensis en Pinus cembroides y $P$. nelsonii (promedio, máximo y mínimo) en Miquihuana, Tamaulipas.

\begin{tabular}{lcc}
\hline & P. cembroides & P. nelsonii \\
\hline No. de inds. $(n)$ & 135 & 46 \\
Promedio (\% \pm 1 D.E.) & $10.6( \pm 8.1)$ & $4.8( \pm 4.5)$ \\
\% Daño Máximo & 40 & 20 \\
\% Daño Mínimo & 1 & 1 \\
Rango & $1-40$ & $1-20$ \\
Ámbito & 39 & 19 \\
Kruskal-Wallis & \multicolumn{2}{c}{227.5} \\
$\left(H-x^{2}\right)$ & $p<0.001$ \\
\hline
\end{tabular}

DE = Desviación estándar, Máx y Mín = valor máximo y mínimo. determinó el reclutamiento de plántulas en cada uno de los cuadrantes por sitio, donde se evaluó el número y altura de las plántulas. Para determinar posibles diferencias entre cuadrantes, se utilizó la prueba de Kruskal-Wallis $\left(\mathrm{H}-\mathrm{Chi}^{2}\right)$ con el programa estadístico PAST versión 2.15 (Hammer et al. 2001). Consecutivamente, se determinó la participación porcentual (PP) de la regeneración natural con respecto a los adultos de cada sitio y de cada especie, utilizando la siguiente fórmula:

$$
P P=\frac{\text { Plántulas }}{\text { Arbolado adulto }} \times 100
$$

\section{RESULTADOS}

Daño por Retinia arizonensis. De Pinus cembroides se registraron un total de 136 plantas, de las cuales 135 presentaron algún porcentaje de daño por $R$. arizonensis. De $P$. nelsonii se contabilizaron 47 plantas, de las que 46 exhibieron cierta proporción de daño de $R$. arizonensis.

Pinus cembroides presentó un daño promedio por Retinia arizonensis en brotes terminales de $10.5 \%( \pm 8.1$ D.E.). En relación a $P$. nelsonii, el porcentaje de daño promedio fue de $4.8 \%( \pm 4.5)$, esto es, ca 2 veces menos que $P$. cembroides (Cuadro 1). Al realizar la prueba de Kruskal-Wallis $\left(H-C h i^{2}\right)$ del porcentaje de ataque de $R$. arizonensis entre $P$. cembroides y $P$. nelsonii, se observó marcada diferencia significativa $(p<0.001)$. De igual manera, al aplicar la misma prueba entre los cuadrantes de cada especie, tanto $P$. cembroides como $P$. nelsonii mostraron diferencias significativas $(p<0.001$ y $P=0.016$, respectivamente), lo cual indica que el daño de $R$. arizonensis difieren entre los cuadrantes de cada especie y entre especies (Cuadro 1).

Respecto al promedio del daño porcentual por $R$. arizonensis, en $P$. cembroides fue ca 2 veces más que en $P$. nelsonii pero el número de plantas en $P$. cembroides se distribuye en todas las categorías de daño, siendo mayor

Cuadro 2. Número de plantas por clases de daño por Retinia arizonensis en Pinus cembroides y P.nelsonii en Miquihuana, Tamaulipas.

\begin{tabular}{|c|c|c|c|c|c|c|}
\hline \multirow[b]{2}{*}{ Clase } & \multirow[b]{2}{*}{ Rango (\%) } & \multirow[b]{2}{*}{ CC } & \multicolumn{2}{|c|}{ Pinus cembroides } & \multicolumn{2}{|c|}{ Pinus nelsonii } \\
\hline & & & No. de árboles & FR\% & No. de árboles & FR\% \\
\hline 1 & $1.0-6.6$ & 3.8 & 57 & 0.42 & 38 & 0.83 \\
\hline 3 & $12.1-17.7$ & 14.9 & 15 & 0.11 & 4 & 0.09 \\
\hline 4 & $17.7-23.3$ & 20.5 & 8 & 0.06 & 1 & 0.02 \\
\hline 6 & 28.9-34.4 & 31.6 & 6 & 0.04 & 0 & 0.00 \\
\hline \multirow[t]{2}{*}{7} & $34.4-40.00$ & 37.2 & 2 & 0.02 & 0 & 0.00 \\
\hline & Totales & & 135 & 1.00 & 46 & 1.00 \\
\hline
\end{tabular}

$\mathrm{CC}=$ centro de clase, $\mathrm{Fr}=$ Frecuencia relativa. 
en la clase 1 con 57 plantas, seguido de la clase 2 con 41 plantas y la clase 3 con 15 plantas que, en su conjunto, representa ca $83 \%$ del total de plantas y agrupa niveles de daño de 1 al $17.7 \%$ por este insecto; el resto de las plantas se integra en las clases 4, 5, 6 y 7 con daño que oscila entre $18 \%$ a $40 \%$. En $P$. nelsonii el comportamiento del daño por $R$. arizonensis fue distinto, ya que la mayoría de los individuos presentaron un daño mínimo (clase $1: 1-6.6 \%)$ con ca $83 \%$ y el resto de las plantas (17\%) se ubicaron en las clases 2, 3 y 4 con daños que oscilan entre 6.6-23.3; no se encontraron plantas con daños superiores al 23.3\% (Cuadro 2).

Densidad y estructura. El arbolado adulto de Pinus cembroides agrupó un total de 136 individuos, en tanto que para $P$. nelsonii fueron 47 individuos.

Referente a la estructura, $P$. cembroides presentó los siguientes valores promedio ( \pm 1 D.E.): diámetro de tallo de $10.7 \mathrm{~cm} \mathrm{(} \pm 8.0)$, altura de $2.9 \mathrm{~m}( \pm 1.0)$ y cobertura de $3.0 \mathrm{~m}^{2}( \pm 1.8)$. En relación a $P$. nelsonii, estos valores fue- ron: diámetro de $10.7 \mathrm{~cm}( \pm 4.3)$, altura de $3.6 \mathrm{~m}( \pm 0.8)$ y cobertura $4.2 \mathrm{~m}^{2}( \pm 1.8)$.

Al compararse los promedios del diámetro y la altura en las dos especies, se aprecian valores muy cercanos, siendo marginalmente superiores para $P$. nelsonii. Sin embargo, para la variable cobertura $P$. nelsonii resultó $c a$ 1.5 veces mayor a $P$. cembroides. Al realizar la prueba de Kruskal-Wallis $\left(\mathrm{H}-\mathrm{Chi}^{2}\right)$ sobre las variables estructurales de las dos especies, se obtuvo una amplia diferencia estadística $(p<0.001)$, incluso dentro de cada especie, excepto para la variable cobertura de $P$. cembroides que fue de $p=0.133$; el resto de las variables presentaron diferencias significativas (Cuadro 3).

El Cuadro 3 y la Figura 1 presentan los promedios, desviaciones estándar, el rango, así como los valores máximos y mínimos de los parámetros estructurales. Es notable, que el ámbito del diámetro de tallo de P. cembroides es superior a $P$. nelsonii, ya que se distribuye en las siete categorías, caso contrario ocurrió en $P$. nelsonii

Cuadro 3. Promedio máximo y mínimo de las variables estructurales de Pinus cembroides y P. nelsonii en Miquihuana, Tamaulipas.

\begin{tabular}{|c|c|c|c|c|}
\hline \multicolumn{2}{|l|}{ Especie } & Diámetro (cm) & Altura (m) & Cobertura( $\left.\mathbf{m}^{2}\right)$ \\
\hline \multirow[t]{4}{*}{ Pinus cembroides $\mathrm{n}=136$} & Promedio $( \pm \mathrm{DE})$ & $10.7( \pm 8.0)$ & $2.9( \pm 1.0)$ & $3.0( \pm 1.8)$ \\
\hline & \% Máx & 39.0 & 6.0 & 8.0 \\
\hline & Rango & $2-39$ & $1-6$ & $0.8-8$ \\
\hline & Ámbito & 37.0 & 5.0 & 7.2 \\
\hline \multirow{3}{*}{ Pinus nelsonii $\mathrm{n}=47$} & \% Mín & 2 & 1.8 & 0.6 \\
\hline & Rango & $2-22$ & $1.8-5$ & $0.6-8$ \\
\hline & Ámbito & 20.0 & 3.2 & 7.4 \\
\hline \multicolumn{2}{|c|}{ Kruskal-Wallis } & 269.9 & 215.3 & 153.1 \\
\hline
\end{tabular}

n = número de individuos, Máx y Mín = valor máximo y mínimo.

Cuadro 4. Número de plantas de cada clase de diámetro de Pinus cembroides y P. nelsonii de Miquihuana, Tamaulipas.

\begin{tabular}{|c|c|c|c|c|c|c|}
\hline \multirow[b]{2}{*}{ Clase } & \multirow[b]{2}{*}{ Rango (cm) } & \multirow[b]{2}{*}{ CC } & \multicolumn{2}{|c|}{ Pinus cembroides } & \multicolumn{2}{|c|}{ Pinus nelsonii } \\
\hline & & & No. de árboles & FR\% & No. de árboles & FR\% \\
\hline 1 & $2.0-7.3$ & 4.6 & 61 & 0.45 & 9 & 0.19 \\
\hline 3 & $12.6-17.9$ & 15.2 & 11 & 0.08 & 12 & 0.25 \\
\hline 4 & 17.9-23.1 & 20.5 & 15 & 0.11 & 3 & 0.06 \\
\hline \multirow[t]{2}{*}{7} & 33.7-39.0 & 36.4 & 4 & 0.03 & 0 & 0.00 \\
\hline & Totales & & 136 & 1.00 & 47 & 1.00 \\
\hline
\end{tabular}

$\mathrm{CC}=$ centro de clase, $\mathrm{Fr}=$ Frecuencia relativa . 


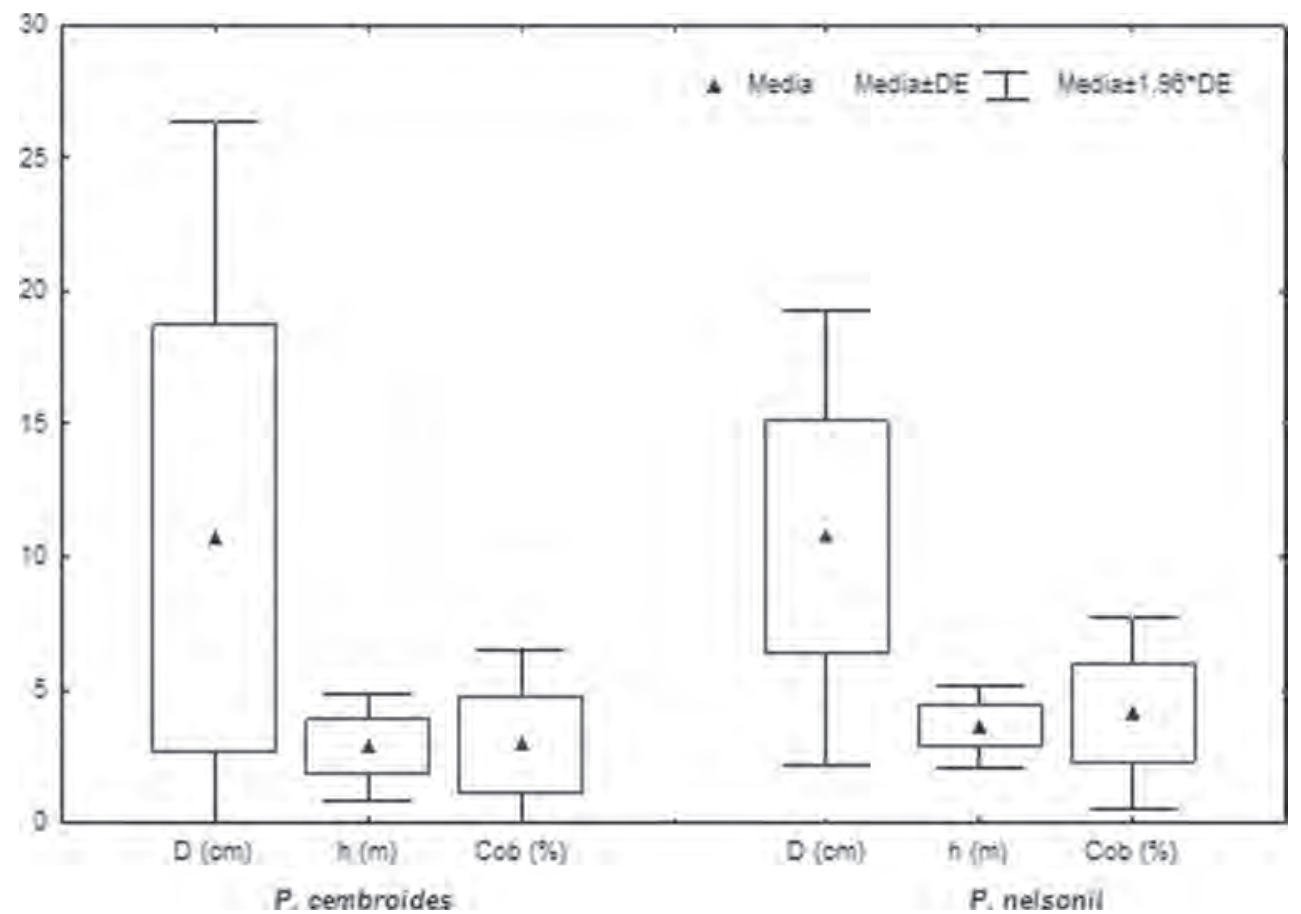

Figura 1. Promedios y desviación estándar de las variables estructurales: diámetro de tallo (D), altura (h) y cobertura (Cob) de Pinus cembroides y P. nelsonii de Miquihuana, Tamaulipas.

donde los diámetros se concentran en las primeras cuatro clases (Cuadro 4).

Si bien, $P$. cembroides contiene individuos de todas las categorías diamétricas de tallo (Cuadro 4 y Figura 2), el número de plantas se concentra en las clases 1 y 2, presentando un $45 \%$ y $28 \%$ respectivamente, que comprende tallos de 2 a $12.6 \mathrm{~cm}$ de diámetro; el otro porcentaje lo integran las clases 3, 4, 5, 6 y 7 con diámetros de tallo de $12.6 \mathrm{~cm}$ a $39.0 \mathrm{~cm}$. Respecto a $P$. nelsonii, su población se agrupa en las primeras cuatro clases, siendo las clases 2 y 3 las más altas, representando un $49 \%$ y $25 \%$, respectivamente, e integrando diámetros de $7.3 \mathrm{~cm}$ a $17.9 \mathrm{~cm}$. El resto de los individuos, se agrupan en las clases 1 y 4 con un $19 \%$ y $6 \%$, respectivamente, presentando diámetros de

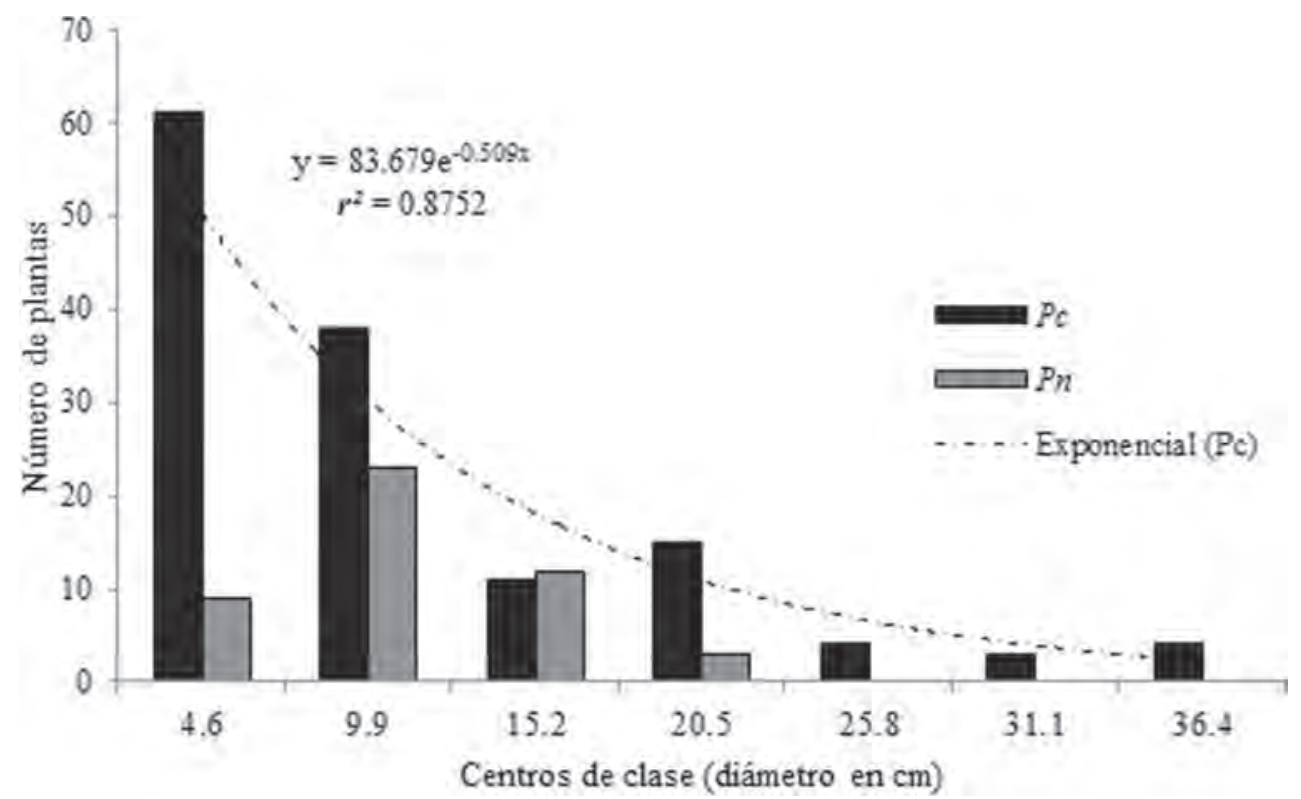

Figura 2. Número de plantas por clase de diametro en Pinus cembroides (Pc) y Pinus nelsonii(Pn) de Miquihuana, Tamaulipas. 
tallo de 2 a $7.3 \mathrm{~cm}$ para la clase 1 , y 17.9 a $23.1 \mathrm{~cm}$ para la cuarta clase (Cuadro 4).

Asociación de las variables estructurales con el daño porcentual de Retinia arizonensis: Pinus cembroides mostró una correlación positiva marginal con las variables altura, diámetro y cobertura de tallo, presentando valores de $r^{2} 0.12,0.16$ y 0.18 , respectivamente $(p<0.001)$. Sin embargo, $P$. nelsonii no manifestó una correlación significativa entre el daño por $R$. arizonensis y los parámetros estructurales, obteniendo valores de $\sim r^{2} 0.05$ y $\sim 0.03$ de las variables diámetro de tallo y cobertura, respectivamente (Cuadro 5).

Respecto a la influencia conjunta de las tres variables estructurales (correlación múltiple $=r^{2}$ ) para cada especie sobre el daño de $R$. arizonensis quedó mayormente explicada en $P$. cembroides con valor de $r^{2}=0.19$ siendo altamente significativa $(p<0.001) \mathrm{y}$, para $P$. nelsonii la influencia de las variables estructurales sobre el porcentaje de daño de $R$. arizonensis no fue significativo ( $p=$ 0.128 ) con valor de $r^{2}=0.12$.

Regeneración de plántulas: Se contabilizaron 28 plántulas de Pinus cembroides y 20 de $P$. nelsonii, siendo la participación porcentual (con respecto a los adultos), de $20.6 \%$ para $P$. cembroides y $42.5 \%$ para $P$. nelsonii. Las plántulas de $P$. cembroides presentaron una altura promedio de $13.9 \mathrm{~cm}( \pm 12.6)$. En $P$. nelsonii, la altura promedio fue de $28.4 \mathrm{~cm}( \pm 20.0)$. Las alturas máximas fueron de $70 \mathrm{~cm}$ y $75 \mathrm{~cm}$ en $P$. cembroides y $P$. nelsonii, respectivamente, y las mínimas de $5 \mathrm{~cm}$ en $P$. cembroides y 10 cm en $P$. nelsonii. Al realizar la prueba de Kruskal-Wallis $\left(\mathrm{H}-\mathrm{Chi}^{2}\right)$ sobre la variable altura de plántulas, se encontró marcada diferencia significativa $(p<0.001)$ entre ambas especies. Similarmente, dentro de cada especie se encontraron diferencias significativas $=p<0.001$ (Cuadro 6).

\section{DISCUSIÓN}

Daño por Retinia arizonensis: De los 137 individuos registrados de $P$. cembroides ca $99 \%$ presentaron diferen-
Cuadro 6. Análisis estadístico de la variable altura de las plántulas de Pinus cembroides y Pinus nelsonii de Miquihuana, Tamaulipas.

\begin{tabular}{lcc}
\hline Estadístico & P. cembroides & P. nelsonii \\
\hline No. de Individuos & 28 & 20 \\
Promedio (cm \pm 1 D.E.) & $13.9( \pm 12.6)$ & $28.4( \pm 20.0)$ \\
Altura Máxima & 70 & 75 \\
Altura Mínima & 5 & 10 \\
Rango & $5-70$ & $10-75$ \\
Ámbito & 65 & 65 \\
Kruskal-Wallis & \multicolumn{2}{c}{71.26} \\
$\left(H-x^{2}\right)$ & \multicolumn{2}{c}{$p<0.001$} \\
\hline
\end{tabular}

tes niveles de daño por $R$. arizonensis. Para $P$. nelsonii el comportamiento del daño es similar a $P$. cembroides, debido a que del total de individuos (47), sólo uno se encontró en estado sano y ca 98\% mostró algún nivel de daño. Aun cuando las dos especies mostraron daño por $R$. arizonensis, para $P$. cembroides el arbolado dañado (83\%) se concentró en las primeras tres categorías (de 1 a $17.7 \%$ ). Para $P$. nelsonii este mismo porcentaje de daño (83\%) se agrupó en la categoría 1 (de 1 a 6.6\%) (Cuadros 1 y 2).

Resultados obtenidos por otros investigadores, sobre el daño que este insecto causa sobre las especies piñoneras muestran lo siguiente: $i$ ) los bosques piñoneros ( $P$. cembroides) de Saltillo, Coahuila, México, presentaron $80 \%$ de daño, causando una mortalidad de ca $50 \%$ (Flores \& Morales 1985), ii) en la misma región, los ataques severos y consecutivos de $R$. arizonensis adicionales a otros agentes nocivos (e.g. plagas, sequías) causaron la muerte del $36 \%$ de las plantas bajo un sistema de plantación comercial (Domínguez 2003), iii) otro hallazgo sobre el detrimento que esta palomilla causa sobre $P$. cembroides, es el registrado en Cardonal, Hidalgo, donde $55.6 \%$ de las ramillas terminales de los piñoneros presentaron mortalidad (Morales 2013). Estos resultados son contrastantes con los obtenidos en el presente estudio, donde los niveles de daño fueron desde 1.2 hasta 42 veces más, respecto a los sitios mencionados (Cuadro 7).

Cuadro 5. Asociación de variables estructurales con el porcentaje de daño por R. arizonensis en Pinus cembroides y Pinus nelsonii de Miquihuana Tamaulipas.

\begin{tabular}{|c|c|c|c|c|c|c|c|}
\hline \multirow[t]{2}{*}{ Especie } & \multirow[t]{2}{*}{ Variable } & \multirow[t]{2}{*}{ No. de Individuos } & \multicolumn{4}{|c|}{ Estadístico } & \multirow{2}{*}{$\begin{array}{c}r^{2} \\
\text { Múltiple }\end{array}$} \\
\hline & & & $r$ & $r^{2}$ & $F$ & $\boldsymbol{P}$ & \\
\hline \multirow[t]{2}{*}{ P. cembroides } & Altura & & 0.3467 & 0.120 & 18.1 & $<0.001$ & \multirow{2}{*}{$\begin{array}{l}r^{2}=.191 \\
p<0.001\end{array}$} \\
\hline & Cobertura & & 0.4310 & 0.186 & 30.34 & $<0.001$ & \\
\hline \multirow[t]{2}{*}{ P. nelsonii } & Altura & & 0.0031 & 0.000 & 0.000 & 0.983 & \multirow{2}{*}{$\begin{array}{l}r^{2}=.125 \\
p<0.128\end{array}$} \\
\hline & Cobertura & & 0.1637 & 0.027 & 1.212 & 0.277 & \\
\hline
\end{tabular}


Cuadro 7. Algunos estudios sobre daños de Retinia arizonensis en pinos piñoneros.

\begin{tabular}{llll}
\hline Especie & Nivel de daño & Lugar & Referencia \\
\hline P. cembroides & 80\% de plantas infestadas & Saltillo, Coah. & Flores \& Morales (1985) \\
y $50 \%$ de mortalidad & & Flores \& Martínez (1987) \\
Pinus cembroides & $2.1 \%$ muerte de los conos & Cardonal, Hgo. & Domínguez (2003) \\
& $\begin{array}{l}\text { 36\% de Mortalidad por ataques } \\
\text { consecutivos de } \text { R. arizonensis } \\
\text { asociados a otros agentes nocivos }\end{array}$ & Ejido Carneros, Saltillo, Coah. & \\
P. cembroides & $\begin{array}{l}\text { 55.6\% de árboles con muerte } \\
\text { de ramillas }\end{array}$ & Cardonal Hgo. & Morales (2013) \\
P. cembroides & $99 \%$ de plantas infestadas & Miquihuana, Tam. & Presente estudio \\
P. nelsonii & $98 \%$ de plantas infestadas & & \\
\hline
\end{tabular}

Densidad y estructura: La densidad encontrada para $\mathrm{Pi}$ nus cembroides fue de 136 individuos (ca 3 veces más respecto a $P$. nelsonii con 47 ), esta especie mostró una marcada uniformidad en todas las categorías diamétricas de los tallos. En contraste con P. nelsonii, la distribución diamétrica se ubicó en las primeras cuatro clases, exhibiendo menores valores de rango, ámbito y desviación estándar (Cuadros 3 y 4).

De acuerdo a las clases diamétricas, en las dos especies se consideran bosques jóvenes ya que concentran su población en las primeras clases diamétricas, presentando forma de " $J$ invertida" en $P$. cembroides (Figura 2). Estas discrepancias categóricas, observadas principalmente en $P$. nelsonii, posiblemente se deban a la presencia de factores adversos como las sequías y la colecta excesiva de semillas; resultados similares fueron obtenidos en $P$. nelsonii, $P$. cembroides y P. pinceana (Suzán et al. 2001 y 2003). Igualmente, en las categorías diamétricas $>23.1$ $\mathrm{cm}, P$. nelsonii está ausente, éste es un factor ecológico que en el futuro podría limitar su regeneración y, por lo tanto, la deriva genética, afectando la adaptación y el éxito de la especie. Este aspecto se constata con la nominación de $P$. nelsonii como especie en peligro de extinción por la Norma Oficial Mexicana NOM-059-SEMARNAT2010 (DOF 2010) y como vulnerable según criterios de la Unión Internacional para la Conservación de la Naturaleza (UICN 2011). En contraposición, P. cembroides que, aún cuando el porcentaje de plantas es bajo (8.1\%) en esta categoría, mantiene árboles en todas las categorías diamétricas, lo cual fundamenta y expresa que la adaptación al medio está regida por una sucesión multigeneracional, donde la posibilidad del éxito reproductivo está garantizado y la especie no presenta riesgos mediatos de supresión ecológica (Cuadro 4 y Figura 2).

Asociación del daño por Retinia arizonensis con las variables estructurales. Respecto a la influencia de las variables dasométricas sobre el daño por $R$. arizonensis, a través de la determinación $\left(r^{2}\right)$, en $P$. cembroides fue significativo $(p<0.001)$ con una correlación positiva marginal con las variables altura, diámetro de tallo y cobertura, presentando valores de $0.12,0.16$ y 0.18 , respectivamente. En $P$. nelsonii la influencia de las variables estructurales sobre el daño no fue significativa, sin embargo, esta especie presentó ligera influencia en el diámetro de tallo y la cobertura. En el efecto combinado de las tres variables estructurales sobre el porcentaje de daño por $R$. arizonensis en $P$. cembroides mediante la regresión múltiple $\left(r^{2}\right)$ se obtuvo un valor de 0.19 , siendo altamente significativa ( $p<0.001$ ), lo cual indica que ca $20 \%$ de la varianza en el daño de $R$. arizonensis es dependiente de las características estructurales de estos bosques. Por su parte, en P. nelsonii la influencia de las variables estructurales sobre el porcentaje de daño de $R$. arizonensis no fue significativa ( $p=0.128$ ) con valor de $r^{2}$ correlación múltiple de 0.12 . Los resultados obtenidos en el presente estudio difieren de lo obtenido por Domínguez (2003), quien encontró que al aumentar el diámetro de $P$. cembroides disminuía el ataque por Retinia y que el mayor daño observado fue en árboles con diámetros $\leq 4.0 \mathrm{~cm}$. En la presente investigación, los valores máximos de daño encontrados en $P$. cembroides ocurrieron en individuos con tallos de $20 \mathrm{~cm}$ y con 3-3.5 m de altura; el individuo con menor daño medía $2.5 \mathrm{~cm}$ de diámetro y $1.80 \mathrm{~m}$ de altura. En P. nelsonii el individuo que presentó mayor daño por $R$. arizonensis (20\% del follaje), exhibió un diámetro de tallo de $14 \mathrm{~cm}$ y $4 \mathrm{~m}$ de altura, en tanto que el árbol con menor daño presentó diámetro de tallo de $\sim 8$ a $12 \mathrm{~cm}$ con altura y de $\sim 3$ a $4 \mathrm{~m}$. Lo anterior puede deberse a que el estudio de Domínguez (2003) se realizó en una plantación con factores controlados como la edad, distancia entre plantas y riego. El presente estudio se realizó bajo condiciones naturales, donde se involucran otras variables diferenciales, tales como la edad de planta, competencia intra e interespecífica y el microclima, entre otros. El mayor daño 
encontrado en $P$. cembroides (ca 2 veces mayor) puede deberse - entre otras causas - a que esta especie presenta mayor densidad, lo cual incrementa la posibilidad de la interacción planta-insecto, lo que queda de manifiesto con lo expuesto por Feeny (1975 y 1976; Lawton, 1983). Así mismo, la expresión de mayor cobertura (mayor fronda) de esta especie respecto a $P$. nelsonii, ha sido documentada anteriormente para bosques templados por Root (1973) y Haddad (2001).

Regeneración. El éxito de cualquier especie está basado en la regeneración natural, la que se mide por la capacidad reproductiva (producción media de semillas), que depende de la correcta polinización, amarre de frutos y la dispersión de semillas. Posteriormente, la predación prey postdispersión, viablidad de la semilla, germinación, crecimiento de las plántulas y el crecimiento vegetativo hasta alcanzar la etapa juvenil (Pardos et al. 2012). Todas estas fases requieren de una serie de factores ambientales, clima y suelo (Pérez-Ramos, 2007; Mostacedo y Pinard, 2001; Queenborodgh et al. 2007) que garanticen el éxito reproductivo. Respecto a este tema, Suzán et al. (2001) y Sánchez-Ramos et al. (2005) señalan que la regeneración natural constituye uno de los problemas torales para la sucesión de las especies piñoneras. En el presente estudio se encontraron 28 plántulas de Pinus cembroides y 20 de $P$. nelsonii en los sitios de estudio. La proporción de plántulas/adultos fue de 20.6 y $42.6 \%$, respectivamente, para estas especies. Estudios anteriores sobre esta variable en la misma zona de estudio fueron realizados por FernandoLuis et al. (2012), donde la regeneración porcentual con respecto a los adultos fue de $49.6 \%$ para $P$. cembroides y 63.4\% para $P$. nelsonii. La óptima regeneración observada para ambas especies, puede deberse entre otros factores al buen contenido de materia orgánica (10.3\% en el sitio con P. cembroides y $8.6 \%$ en el sitio con P. nelsonii), con lo que se clasifican como suelos ricos (Salgado et al. 2006) lo que mejora la captación de humedad que es uno de los requisitos básicos para la germinación (Mostacedo \& Pinard 2001, Pérez-Ramos 2007, Queenborodgh et al. 2007).

Pinus nelsonii presenta una estructura vertical y horizontal poco compacta, con ramificación más abierta y una densidad de plantas adultas menor. Por otra parte $P$. cembroides, al mostrarse con una estructura densa y copiosa, aumenta la competencia por luz y nutrientes que también son elementales en la germinación (Mostacedo \& Pinard 2001, Pérez-Ramos 2007, Queenborodgh et al. 2007). Además, algunos autores mencionan que el incremento de la temperatura por el cambio climático mejora la regeneración natural, sobre todo en las regiones frías (Fenner y Thompson 2005). Sin embargo, estos resultados son supuestos ya que estas plántulas pueden ser afectadas por las sequías, la competencia por agua y nutrientes, así como daños por el pastoreo como lo señalan Calama \& Montero (2007) en Pinus pinea (L).

Es indudable la necesidad de complementar los resultados sobre el daño de Retinia arizonensis, así como los factores que favorecen el ataque de pinos piñoneros en la región de Miquihuana, debido a que el porcentaje de plantas (ca 99\% en P. cembroides y ca $98 \%$ en P. nelsonii) con daños de diversa magnitud, supera a lo reportado por otros investigadores. Así mismo, aun cuando los daños no provocan la muerte inmediata de las plantas, retardan el crecimiento vegetativo y en consecuencia, afectan la producción de conillos y conos, reduciendo por ende la producción de semillas, lo que en el futuro afectará la regeneración natural de estos pinos piñoneros.

AGRADECIMIENTOS. Al Conacyt, por la Beca otorgada al primer autor (IFL) para realizar estudios de Doctorado. Al Comité de Tesis Doctoral del Instituto de Ecología Aplicada, Universidad Autónoma de Tamaulipas: Gerardo Sánchez-Ramos (Director de Tesis y Asesor), José Guadalupe Martínez-Ávalos (Coasesor), Humberto Suzán-Azpiri (Coasesor) y José Villanueva-Díaz (Coasesor). A la Dirección General de Educación Tecnológica Agropecuaria dependiente de la Subsecretaría de Educación Media Superior, por el permiso otorgado al primer autor (IFL) para realizar estudios de Doctorado. Al Ing. Manuel de Jesús Yáñez-Pacheco y al Sr. Guillermo Martínez Hernández, por su valiosa ayuda en el trabajo de campo. A los revisores, por los comentarios y sugerencias realizadas a este manuscrito.

\section{LITERATURA CITADA}

Adams, H. D., Guardiola-Claramonte, M., Barrón-Gafford, G. A., Villegas, J. C., Breshears, D. D., Zoug, C. B., Troch, P. A. \& Huxman, T. E. 2009. Temperature sensitivity of drought-induced tree mortality portends increased regional die-off under global-change-type drought. Proceedings of the National Academy of Sciences, 106: 7063-7066.

Basáñez-Muñoz, A. 1983. Contribución al Conocimiento de la Ecología de los Bosques de Pino Piñonero (Pinus cembroides y Pinus nelsonii) en el Municipio de Miquihuana, Tamaulipas. Tesis profesional (Biólogo). Escuela de Ciencias Biológicas, Universidad del Noreste. México. 110 pp.

Calama, R. \& Montero, G. 2007. Cone and seed production from stone pine (Pinus pinea L.) stands in Central Range (Spain). European Journal of Forest Research, 126: 23-35.

Caribello, J. 2003. Restauración de ecosistemas a partir del manejo de la vegetación. Guía metodológica Ministerio de Ambiente, Vivienda y Desarrollo Territorial. Colombia. 96 pp.

Challenger, A. 1998. Utilización y Conservación de los Ecosistemas Terrestres de México. Pasado, Presente y Futuro. (Ed.) CONABIO, Instituto de Biología, UNAM, Agrupación Sierra Madre, S.C. México. 847 pp.

Cibrián-Tovar, D. 1985. Insectos de los Piñoneros, pp. 174-192. In: Flores, L. J., Cantú, A. C. M. \& Marroquín, J. (Eds.). I Simposium Nacional sobre Pinos piñoneros, Facultad de Silvicultura. U.A.N.L Linares, N. L. México. 
Cibrián-Tovar, D., Méndez, M. T., Campos, B. R., Yates III, H. O. \& Flores, L. J. 1995. Insectos Forestales de México. Editorial Madero. Universidad Autónoma Chapingo. Secretaría de Agricultura y Recursos Hidráulicos, USDA Forest Servise. Natural Resources Canada y Comisión Forestal de América del Norte FAO. Pub. No. 6. 453 pp.

Cibrián T., D. \& Méndez, T. 1987. Manejo de plagas forestales en la producción de piñones, pp. 111-121. In: Marie-Francoise Passini y David Cibrián Tovar (Eds.). II Simposio Nacional sobre Pinos piñoneros, Memorias. Ed. UANL. México

CONAFOR. 2013. Informes anuales. Estadística Final Forestales: incendios forestales. http://www.conafor.gob.mx/portal/index.php/ temas-forestales/incendios

Cranshaw, W. S., Leatherman, D. A., Jacobi ,W. R. \& Mannix, L. 2000. Insects and diseases of woody plants of the central Rockies. Bulletin 506A. Fort Collins, CO: Colorado State University, Cooperative Extension. 283 pp.

Currano, E. D., Wilf, P., Wing, S. L., Labandeira, C. C., Lovelock, E. C. \& Royer, D. L. 2008. Sharply increased insect herbivory during the Paleocene-Eocene Thermal Maximum. Proceedings of the National Academy of Sciences, 105(6): 1960-1964.

DeLucia, E. H., Casteel, C. L., Nabity, P. D. \& O’Neill, B. F. 2008. Insects take a bigger bite out of plants in a warmer, higher carbon dioxide world. Proceedings of the National Academy of Sciences, 105: 1781-1782.

Díaz-Ortiz, B. E. 1988. Insectos que afectan conos y semillas en la Sierra de San Pedro Mártir, Baja California. Ciencia forestal: Revista del Instituto Nacional de Investigaciones Forestales, 13: 29-70.

Diario oficial de la federación (DOF). 2010. Norma Oficial Mexicana NOM-059-SEMARNAT-2010, Protección ambiental-Especies nativas de México de flora y fauna silvestres-Categorías de riesgo y especificaciones para su inclusión, exclusión o cambio-Lista de especies en riesgo. Disponible en: http://www.profepa.gob.mx/innovaportal/file/435/1/NOM_059_SEMARNAT_ 2010.pdf.

Domínguez, C. G. 2003. Evaluación del daño causado por Retinia arizonensis, Miller (Lepidoptera:Torticidae), en una plantación de Pinus cembroides, Zucc, en el Ejido Carneros, Saltillo, Coahuila. Tesis de Licenciatura de Ingeniero Forestal. UAAAN, Saltillo, Coahuila. $62 \mathrm{pp}$.

Fenner, M. \& Thompson, K. 2005. The Ecology of Seeds. Cambridge, U.K. Cambridge University Press. 250 pp.

Feeny, P. 1975. Biochemical coevolution between plants and their insect herbivores, pp. 3-19 In: Gilbert L. E. \& Raven, P. H. (Eds.). Coevolution of animals and plants. University of Texas Press, Austin.

Feeny, P. 1976. Plant Apparency and Chemical Defense. Recent Advances in Phytochemistry, 10: 1-40.

Fernando-Luis, I., Sánchez-Ramos, G. \& Martínez-Ávalos, G. 2012. Estructura y regeneración natural de pinos piñoneros (Pinus nelsonii y Pinus cembroides) en el municipio de Miquihuana, Tamaulipas, pp.14 In: Perez-Quilantán \& A. Gatica (Eds). Memorias en Resumen. Expociencia e Innovación Tamaulipas 2012.

Flores L., J. \& Caldera, F. 1985. Características de los conos de algunos piñoneros en relación al ataque de insectos, pp. 223-232 In: Flores L., J., Cantú A., C. M., Marroquín, J. (Eds.). I Simposium Nacional sobre Pinos piñoneros, Facultad de Silvicultura. U.A.N.L. Linares, N.L.

Flores F., J. \& Díaz, D. E. 1991. Evaluación de la mortalidad de conos y semillas en diferentes especies de piñoneros, localizados cerca de Saltillo, Coahuila, pp. 105-110. In: Sánchez, G., Sáenz, C. \& Ledezma, S. (Eds.). IV Simposio Nacional sobre Pinos Piñoneros. Cd. Victoria, Tamaulipas.

Flores, A. J. \& Martínez, R. S. 1987. Contribución al conocimiento de la biología e importancia de algunos insectos que se alimentan de conos y semillas de Pinus cembroides. Tesis profesional UACH. México 186 pp.

Franklin, J. F., Spies, T. A., Pelt, R. V., Carey, A. B., Thornburgh, D. A., Berg, D. R., Lindenmayer, D. B., Harmon, M. E., Keeton, W. S., Shaw, D. C., Bible, K. \& Chen, J. 2002. Disturbances and structural development of natural forest ecosystems with silvicultural implications, using Douglas-fir forests as an example. Forest Ecology and Management, 155: 399-423.

Franklin, J. F. \& Van Pelt, R. 2004. Spatial aspects of structural complexity in old-growth forests. Journal of Forestry, 102: 22-28.

García, E. 1981. Modificaciones al sistema de clasificación climática de Köppen. Instituto de Geografía, Universidad Nacional Autónoma de México, México, D.F.

Gernandt, D. S., Liston, A. \& Piñero, D. 2003. Phylogenetics of Pinus subsections Cembroides and Nelsoniae inferred from cpDNA sequences. Systematic Botany, 28: 657-673.

Gómez-Pompa, A. \& Ludlow, W. B. 1976. "Regeneración de los ecosistemas tropicales y subtropicales”, pp. 11-30. In: GómezPompa, A., Del Amo, S., Vázquez-Yanes, C. \& Butand-Cervera, C. (Eds.). Investigaciones sobre la regeneración de Selvas Altas en Veracruz, México. Continental, S. A. de C. V. México.

Haddad, N. M., Tilman, D., Haarstad, J., Ritchie, M. \& Knops, J. M. 2001. Contrasting effects of plant richness and composition on insect communities: a field experiment. The American Naturalist, 158: 17-35.

Hammer, O., Harper, D. A. T. \& Ryan, P. D. 2001. PAST version 2.15.Paleontological Statics software Package for Education and Data Analysis.Paleontologia Electronica 4: 9.

Hanks, L. 1999. Influence of the larval host plant on reproductive strategies of cerambycid beetles. Annual review of Entomology, 44: 483-505.

Harper, J. L. 1977. Population biology of plants. Academic Press. London, UK. 892 pp.

Instituto Nacional de Estadística y Geografía (INEGI). 2009. Prontuario de información geográfica municipal de los Estados Unidos Mexicanos: Miquihuana, Tamaulipas. México en cifras. Información nacional, por entidad federativa y por Municipios disponible en: http://www3.inegi.org.mx/sistemas/mexicocifras/

Jacobi, W. R. \& W. S. Cranshaw 2009. Pinyon Pine Diseases and Insects Fact Sheet No. 2.948. Gardening Series/Diseases. Colorado State University Extension. USA. pp: 12-14.

Jactel, H., Menassieu, P., Raise, G. \& Burban, C.1996. Sensitivity of pruned maritime pine (Pinus pinaster Ait) to Dioryctria sylvestrella Ratz.(Lep., Pyralidae) in relation to tree vigour and date of pruning. Journal of Applied Entomology, 120: 153-157.

Jactel, H., Goulard, M., Menassieu, P. \& Goujon, G. 2002. Habitat diversity in forest plantations reduces infestations of the pine stem borer Dioryctria sylvestrella. Journal of Applied Ecology, 39: 618-628.

Jentsch, A., Kreyling, J. \& Beierkuhnlein, C. 2007. A new generation of climate-change experiments: Events, not trends. Frontiers in Ecology and the Environment, 5: 365-374.

Jiménez, J., Aguirre, O. \& Kramer, H. 2001. Análisis de la estructura horizontal y vertical en un ecosistema multicohortal de pino-encino en el norte de México. Investigaciones Agrarias en Sistemas y Recursos Forestales, 10: 355-366. 
Lawton, J. H. 1983. Plant architecture and the diversity of phytophagous insects. Annual Review of Entomology, 28: 23-39.

Ledezma, S., Sánchez, G., Suzán, H. \& Flores, J. 1991. Evaluación de la mortalidad y Tabla de vida en conos de Pinus nelsonii Shaw en el municipio de Miquihuana, Tamaulipas, pp. 67-77. In: Sánchez, G., Sáenz, C. \& Ledezma, S. (Eds.), IV Simposio Nacional Sobre Pinos Piñoneros. UAT-COTACYT-UANL, Tamaulipas, México.

Maleque, M. A., Maeto, K., Makino, S. I., Goto, H., Tanaka, H., Hasegawa, M. \& Miyamoto, A. 2010. A chronosequence of understorey parasitic wasp assemblages in secondary broad-leaved forests in a Japanese 'satoyama' landscape. Insect Conservation and Diversity, 3: 143-151

Marquis, R. J. \& Whelan, C. J. 1994. Insectivorous birds increase growth of white oak through consumption of leaf chewing insects. Ecology, 75: 2007-2014.

Martin, K., Norris, A. \& Drever, M. 2006. Effects of bark beetle outbreaks on avian biodiversity in the British Columbia interior: Implications for critical habitat management. BC Journal of Ecosystems and Management, 7: 10-24.

Martínez, R. S., Flores, J. \& Cibrián, D. 1985. Evaluación del daño y plan de manejo de las principales plagas de conos y semillas de piñón en el Estado de Hidalgo, pp. 215-222. In: Flores, L. J., Cantú, A. C. M. \& Marroquín, J. (Eds.) I Simposium Nacional sobre Pinos piñoneros, Facultad de Silvicultura. U.A.N.L. Linares, N.L. México.

Martínez-Ramos, M. \& García-Orth, X. 2007. Sucesión ecológica y restauración de las selvas húmedas. Boletín de la Sociedad Botánica de México, 80: 69-84.

Martínez-Ramos, M. 1994. Regeneración natural y diversidad de especies arbóreas en selvas húmedas. Boletín de la Sociedad Botánica de México, 54: 179-224.

Martínez-Ramos, M. 1995. "Regeneración natural y diversidad de especies arbóreas en selvas húmedas”, pp. 27-79. In: Delfín, G.H., Parra, T. V. \& Echazarreta, G. C. (Eds.) Conocimiento y manejo de las selvas de la Península de Yucatán. Universidad Autónoma de Yucatán. México.

Menéndez, R. 2007. How are insects responding to global warming? Tijdschriftvoor Entomologie, 150: 355-365.

Mitton, J. B. \& Ferrenberg, S. M. 2012. Mountain pine beetle develop an unprecedented summer generation in response to climate warming. American Naturalist, 179: E163-E171.

Morales, B. J. 2013. Propuesta de administración silvícola para la producción de piñón en el ejido La Florida, Cardonal, Hidalgo. Tesis de Licenciatura. UACH. México 63 pp.

Mostacedo, B. \& Pinard, M. 2001. "Ecología de semillas y plántulas de árboles maderables en bosques tropicales de Bolivia”, pp. 1129. In: Mostacedo, B. \& Fredericksen, T. S. (Eds.). Regeneración y silvicultura de bosques tropicales en Bolivia. Proyecto de manejo forestal sostenible (BOLFOR), Santa Cruz, Bolivia.

Mostacedo, B. \& Fredericksen, T. 2000. Manual de Métodos Básicos de Muestreo y Análisis en Ecología Vegetal. Ed. El País, Santa Cruz, Bolivia. 87 pp.

Pardos, M., Bravo, F., Gordo, J., Montero, G. \& Calama, R. 2012. La investigación en regeneración natural de las masas forestales. pp. 17-36. In: Gordo J., Calama, R., Pardos, M., Bravo, F. \& Montero, G. (Eds). La regeneración natural de los pinares en los arenales de la Meseta Castellana.Instituto Universitario de Investigación en Gestión Forestal Sostenible (Universidad de ValladolidINIA).Valladolid, España.

Paz, H. \& Martinez-Ramos, M. 2003. Seed mass and seedling per- formance within eight species of Psychotria (Rubiaceae). Ecology, 84: 439-450.

Pérez-Ramos, I. M. 2007. Patrones de regeneración natural de especies leñosas a nivel de comunidad. Ecosistemas, 16: 131-136.

Perry, D. A., Jing, H., Youngblood, A. \& Oetter, D. R. 2004. Forest Structure and Fire Susceptibility in Volcanic Landscapes of the Eastern High Cascades, Oregon. Conservation Biology, 18: 913926.

Perry, J. 1991. The Pines of Mexico and Central America. Timber Press. Portland, Oregon, USA. 231 pp.

Queenborough, S. A., Burslem, D. F., Garwood, N. C. \& Valencia, R. 2007. Neighborhood and community interactions determine the spatial pattern of tropical tree seedling survival. Ecology, 88: 2248-2258.

Ramírez-Marcial, N., González-Espinosa, M. \& Williams-Linera, G. 2001. Anthropogenic disturbance and tree diversity in montane rain forest in Chiapas, Mexico. Forest Ecology and Management, 154: 311-326.

Redmond, M. D., Forcella, F. \& Barger, N. N. 2012. Declines in pinyon pine cone production associated with regional warming. Ecosphere, 3(12): art120.

Root, R. B. 1973. Organization of a Plant-Arthropod Association in Simple and Diverse Habitats: The Fauna of Collards (Brassica oleracea). Ecological Monographs, 43: 95-124.

Ruiz C. E., Coronado B., J. M., Escamilla G., O. S., Hernández A., S. G. \& Covarrubias D., C. A. 1997 a. Himenópteros colectados en follaje de Pinus cembroides Zucc. y P. nelsonii Shaw en Tamaulipas, México. Biotam, 8: 33-40.

Ruiz C. E., Coronado B., J. M. \& Escamilla G., O. S. 1997 b. Plagas de Pinus cembroides Zucc. y P. nelsonii Shaw en tres municipios de Tamaulipas. Biotam, 8: 41-48.

Salgado-García S., Palma-López, D. J., Lagunes-Espinoza, J. \& Castelán-Estrada, M. 2006. Manual para el muestreo de suelos plantas y aguas e interpretación de análisis. Colegio de Postgraduados, Campus Tabasco-ISPROTAB. H. Cárdenas, Tabasco, México. 90 pp.

Samo, A. J., Garmendia, A. \& Delgado, J. A. 2008. Introducción Práctica a la Ecología. Pearson Educación, S. A. Madrid España. p. 227. ISBN 978-84-8322-445-8.

Sánchez-Castillo, C. 2013. Caracterización del hábitat y estructura poblacional de Pinus pinceana Gordon (Pinaceae) en Tamaulipas, México. Tesis de Licenciatura. Unidad Académica Multidisciplinaria de Ciencias, Educación y Humanidades, UAT. 58 pp.

Sánchez-Ramos, G., S. Ledezma \& H. Suzán. 1987. Estimación de daños por insectos en conos inmaduros de Pinus nelsonii en Miquihuana, Tamaulipas, pp 82-86. In: Flores F., J., Flores L., J., García Moya, E. \& Lira, R. H. (Eds.) Memorias del III Simposio Nacional Sobre Pinos Piñoneros. UAAAN. México.

Sánchez-Ramos, G., Ledezma, S., Suzán, H. \& Flores, J. 1991. Utilización de la Técnica de Análisis de cono aplicada en semillas de Pinus nelsonii Shaw, provenientes de Miquihuana, Tamaulipas, pp. 89-95. In: Sánchez, G., Sáenz, C. \& Ledezma, S. (Eds.). IV Simposio Nacional Sobre Pinos Piñoneros. UAT-COTACYTUANL, Tamaulipas, México.

Sánchez-Ramos, G. \& Ledezma, S. 1995. Los bosques piñoneros de Tamaulipas un recurso potencial para la región semiárida: Estudio de su problemática sanitaria. Acta Cientifica Potosina, 13: 79-102.

Sánchez-Ramos, G., Martínez-Ávalos, J. \& Suzán, H. 2005. Los Pinos Piñoneros, pp. 266-272. In: Sánchez, G., Reyes, P. \& Dirzo, R. (Eds.) Historia Natural de la Reserva de la Biosfera El Cielo. 
Tam., Méx. Inst. de Ecología Alimentos-UAT, Instituto de Ecología, A. C. e Instituto de Ecología-UNAM.

Sánchez-Ramos, G. \& Dirzo, R. 2005. Patrones de herbivoría por Lepidoptera en plantas del bosque mesófilo, pp. 185-191. In: Sánchez-Ramos, G., Reyes-Castillo, P. \& Dirzo, R. (Eds.). Historia Natural de la Reserva de la Biosfera El Cielo. Tam., Méx. Inst. de Ecología Alimentos-UAT, Instituto de Ecología, A. C. e Instituto de Ecología-UNAM.

Secretaría de Desarrollo Urbano y Medio Ambiente (SEDUMA). 2013. Combaten Incendio en Miquihuana. Comunicado de prensa 0378-abril 27, 2013. Disponible en: http://tamaulipas.gob. mx/2013/04/abaten-incendio-en-zona-de-miquihuana/

StatSoft, Inc. 2004. Statistica (data analysis software system), version 6. www.statsoft.com.

Steeghs, M., Bais, H. P., de Gouw, J., Goldan, P., Kuster, W., Northway, M., Fall, R. \& Vivanco, J. M. 2004. Proton-transferreaction mass spectrometry (PTR-MS) as a new tool for real time analysis of root-secreted volatile organic compounds (VOCs) in Arabidopsis thaliana. Plant Physiology, 135: 47-58.

Sturges, H. A.1926. The Choice of a Class Interval. Journal of the American Statistical Association, 21: 65-66. Disponible en: http:// www.jstor.org/stable/2965501 .Accessed: 06/04/2012 02:17

Suzán, H. 1985. Estudios autoecológico-dendrocronológicos en Pinus nelsonii. pp. 137-149. In: Flores, L. J., Cantú A., C. M. \& Marroquín, J. (Eds.). I Simposium Nacional sobre Pinos piñoneros, Facultad de Silvicultura. U.A.N.L. Linares, N.L. México

Suzán, H. 1987. Estudios ecológicos en Pinus nelsonii en Tamaulipas pp. 199-210. Memorias del Segundo Simposio Nacional sobre Pinos Piñoneros. México

Suzán, H. 1990. Estructura de un bosque de Pinus nelsonii Shaw En Tamaulipas. Biotam, 1: 29-35.

Suzán, H., Sánchez, G. \& Ledezma, S. 1991. El cultivo de pinos piñoneros: una alternativa para regiones semiáridas. pp. 57-64. In: G. Sánchez, C. Sáenz y S. Ledesma (Eds.) IV Simposio Nacional
Sobre Pinos Piñoneros. UAT-COTACYT-UANL. Tamaulipas, México.

Suzán, H. \& Galarza, J. 1987. Manejo ecológico de un bosque de pinos piñoneros en Tamaulipas, pp. 164-170 In: Aldon, E., González, C. \& Moir, W. (Eds.). Strategies for classification and management of native vegetation for food production in arid zones. USDA, Technical Reports RM-150.

Suzán, H. \& Sánchez-Ramos, G.1989. Caracterización biótica de una comunidad de Pinus nelsonii Shaw en Tamaulipas, pp. 54-58. In: Flores F., J. Flores L., J., García Moya, E. \& Lira, R. H. (Eds.). Memorias del III Simposio Nacional Sobre Pinos Piñoneros. UAAAN. México.

Suzán-Azpiri, H., Sánchez-Ramos, G., Martínez-Ávalos, J. G., Villa-Melgarejo, S. \& Franco, M. 2001. Population structure of Pinus nelsoni Shaw, an endemic pinyon pine in Tamaulipas, Mexico. Forest Ecology and Management, 165: 193-203.

Suzán-Azpiri, H., Sánchez-Ramos, G. \& Enríquez, G. 2003. Estructura poblacional y sanidad forestal en bosques de Pinos piñoneros en el estado de Querétaro, México. BioTam Nueva Serie, 14: $1-12$

UICN (Unión Internacional para la Conservación de la Naturaleza). 2011. IUCN Red List of Threatened Species. Version 2011.2.<www.iucnredlist.org>. Downloadedon24 November 2011.

Urbieta, I. 2009. Estructura, dinámica y regeneración de los bosques mixtos de alcornoque (Quercus suber L.) y quejigo moruno ( $Q$. canariensis Willd.) del sur de la Península Ibérica: una aproximación multiescala. Ecosistemas, 18:116-121.

Wenninger, E. J. \& Inouye, R. S. 2008. Insect community response to plant diversity and productivity in a sagebrush-steppe ecosystem. Journal of Arid Environments, 72: 24-33.

Wold, E. N. \& Marquis, R. J. 1997. Induced defense in white oak: Effects on herbivores and consequences for the plan. Ecology, 78: 1356-1369. 